
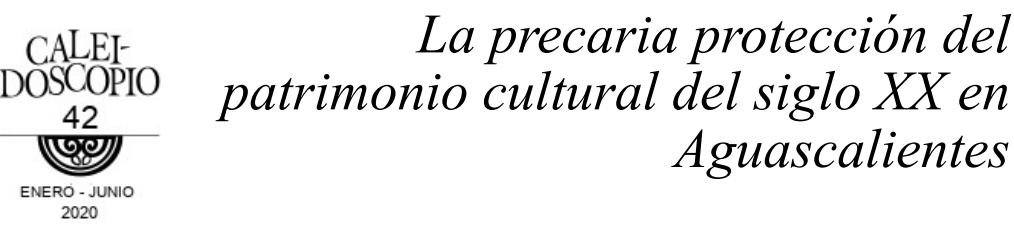

The precarious protection of the cultural heritage of the twentieth century in Aguascalientes

JOSÉ JUAN HERNÁNDEZ MONCADA Universidad de las Artes, México

RESUMEN

En el presente artículo se presentará un análisis y reflexión general sobre la legislación vigente en materia de protección al patrimonio cultural tangible y las consecuencias que dicho marco legal tiene sobre la precaria o nula protección sobre bienes de reciente creación, concretamente una gran cantidad de inmuebles de gran valor que existen en la ciudad de Aguascalientes y que actualmente no gozan de protección de ninguna ley ni institución.

Palabras clave: Patrimonio cultural; legislación cultural mexicana; Aguascalientes; patrimonio del siglo XX; arquitectura moderna.

\title{
ABSTRACT
}

The following article an analysis and general reflection will be presented on current legislation on the protection of 
tangible cultural heritage and the consequences that this legal framework has on the precarious or null protection over newly created assets, specifically a large number of high-value properties that exist in the city of Aguascalientes and that are not protected by any law or institution.

Keywords: Cultural heritage; mexican cultural law; Aguascalientes; twentieth century heritage; modern architecture.

El patrimonio cultural en el estado de Aguascalientes se compone por bienes a los cuales se les pueda atribuir un valor o interés cultural, dichos bienes pueden ser de carácter material o inmaterial. La definición del patrimonio cultural se vuelve más compleja cuando revisamos los criterios aplicables para atribuir valor o interés cultural a determinado bien, ya que cultura no es un concepto único, absoluto, ni estático; pero sin entrar en demasiados detalles en las diversas acepciones de la palabra cultura, sabemos que aquello que tiene valor para una sociedad; tiene valor en tanto esos bienes son testimonio y memoria de los procesos socioculturales e históricos que la sociedad ha atravesado.

Estos procesos son visibles en Aguascalientes especialmente a raíz de la bonanza industrial derivada de la llegada del ferrocarril a finales del siglo XIX. Testimonio de este periodo de industrialización y constantes cambios, son una gran riqueza arquitectónica que engalana nuestras calles, perteneciente a distintas corrientes estilísticas de la edad moderna, como el historicismo arquitectónico, el Art Noveau, el Art Déco, el Funcionalismo entre otros. Por lo 
que podríamos afirmar que la ciudad adquirió su identidad física y cultural durante los últimos años del siglo XIX y gran parte del siglo XX, por lo que la mayoría de las construcciones, creaciones artísticas y monumentos datan de ese periodo.

Sin embargo, el problema que pretende señalar este texto versa entorno a una situación de vulnerabilidad y riesgo de paulatina desaparición de estos bienes; propiciado en mayor medida por factores antrópicos, debido a la carencia de una legislación adecuada en la materia, ya que la norma actualmente vigente se encuentra plagada de definiciones ambiguas y vacíos de autoridad que impiden la adecuada, detección, catalogación, protección y gestión de dichos bienes que forman parte del Patrimonio Cultural de Aguascalientes.

Uno de los primeros y mayores retos en materia de patrimonio cultural siempre ha sido su preservación, son muchos los factores y actores que influyen de una u otra manera dentro del rubro, uno de ellos y de vital importancia es sin duda el marco jurídico, el cual es necesario para generar el esquema de protección básico e idóneo para el patrimonio cultural, en claro beneficio de la sociedad, sin embargo legislar en la materia no es una tarea fácil, dado que el mismo concepto de patrimonio cultural resulta ambiguo, por lo tanto es aún mas complejo definirlo y categorizarlo dentro del derecho positivo.

Existen distintos criterios dentro de las normas jurídicas para categorizar el patrimonio cultural, usualmente se suele recurrir a un criterio cuantitativo que es la temporalidad, es decir la fecha de la que data el bien en cuestión, no obstante, el problema comienza cuando la temporalidad se ve rebasada, es decir cuando se trata de bienes que datan del siglo XX, a pesar de que cuenten atributos de interés cultural. La categoría de los bienes 
datados del siglo XX, suele denominarse usualmente dentro de la ley como patrimonio o bienes artísticos; al atribuir un valor artístico y así suele descartarse un criterio temporal, entonces se recurre comúnmente a criterio cualitativo y estético, ante lo cual resulta una tarea titánica, por no imposible, definir un criterio absoluto y a nivel general para que un bien pueda ser considerado dentro de esta categoría de patrimonio. Ya que las realidades a nivel nacional y regional suelen ser diametralmente distintas, por lo cual es extremadamente difícil establecer criterios generalizados.

\section{EL PATRIMONIO}

Antes de continuar describiendo la situación, vale la pena primero comprender responder la pregunta sobre ¿A qué nos referimos cuando hablamos de Patrimonio Cultural? Patrimonio cultural se refiere a aquellas creaciones humanas de cierto valor, divididas por su naturaleza física en patrimonio tangible e intangible, pero en el presente análisis nos centraremos específicamente en el patrimonio tangible.

El término actualmente es por sí trillado; familiar para la gran mayoría de la población, pero su comprensión es mucho más limitada, desde una definición de diccionario patrimonio sería todo bien o conjunto de bienes legados por un antepasado y heredables a un sucesor; en este sentido el patrimonio cultural cumple a cabalidad con esta acepción, ya que constituye el testigo y memoria de un pasado para una generación futura. Sin embargo, el debate se centra en los criterios sobre qué bienes y de qué naturaleza pueden o deben ser considerados como parte de ese patrimonio cultural; por lo que la definición del patrimonio se remite a criterios, los cuales que no 
pueden permanecer estáticos puesto que la cultura y el ser humano se encuentran en constante evolución, derivado de esto sería teóricamente imposible acuñar una definición absoluta e inamovible de lo que representa el patrimonio cultural.

Llull (2005) acuñó una definición, producto de confrontar a distintos teóricos, la cual dice lo siguiente: "Podemos definir el patrimonio cultural como el conjunto de manifestaciones u objetos nacidos de la producción humana, que una sociedad ha recibido como herencia histórica, y que constituyen elementos significativos de su identidad como pueblo." (p. 181)

Partiendo de la definición anteriormente citada, al ser el patrimonio parte inalienable de la identidad de un pueblo, cobra una relevancia mayor ante lo cual se ha generado la imperante necesidad de procurar su conservación y protección en beneficio de las generaciones posteriores; con esta definición la visión general y técnica del patrimonio quedaría adecuadamente descrita; sin embargo para poder significar y valorar adecuadamente el patrimonio cultural hay que contextualizarlo en su entorno social y su aspecto como elemento de identidad para lo cual Carlos Moreno (2019) nos brinda una interesante reflexión en la que equipara al patrimonio con un ente vivo; es decir, al ser el testigo de los procesos de desarrollo social, el patrimonio no permanece inalterado, de lo cual se deriva la naturaleza evolutiva del patrimonio.

Además de la reflexión centrada en los factores de índole social, podemos encontrar otro tipo de reflexión la cual ha sido motivada por acontecimientos históricos que amenazaron gravemente al patrimonio cultural; (especialmente las dos Guerras Mundiales) desde entonces la humanidad ha ido estableciendo medidas para su protección y las medidas más trascendentales y univer- 
sales que el ser humano tiene capacidad de implementar han sido a través del derecho positivo, la formulación de ordenamientos jurídicos; es decir las leyes; las cuales tienen en parte su sustento en los congresos internacionales de patrimonio y monumentos cuyo testimonio nos llega a través de la Carta de Atenas (1931) la Carta Italiana del Restauro (1932) y la Carta de Venecia (1964)

\section{LA LEY FEDERAL}

Un punto de coincidencia la mayoría de los teóricos del estado es que la ley positiva debe ser un ordenamiento racional necesario para garantizar un bien común, desde los clásicos como Platón, pasando por los escolásticos como San Agustín de Hipona o Santo Tomás de Aquino, hasta los teóricos de la edad moderna como Maquiavelo, Hobbes o Rousseau entre otros; coinciden en que estos ordenamientos son necesarios para el bienestar de la sociedad misma; partiendo de esta reflexión el patrimonio cultural al ser un elemento de la identidad y la memoria social, su cuidado y preservación es parte de este bien común, por lo tanto es pertinente hacer una revisión del esquema legislativo vigente que protege nuestro patrimonio a nivel federal y local.

La preocupación del ser humano por proteger su patrimonio cultural tiene raíces antiguas, desde el surgimiento del coleccionismo en la Europa del siglo XV en el cual se rescataban, estudiaban y replicaban las antigüedades; hasta nuestros días con la creación de instituciones y organismos internacionales. En nuestro país el primer antecedente legal respecto a la protección del patrimonio lo encontramos durante el Porfiriato en el año de 1885 con la creación de la primera figura encargada de la protección de bienes culturales: el inspector y 
conservador de monumentos arqueológicos de la República (Cottom, 2008).

Y así en 1896 se promulgó la Ley sobre Exploraciones Arqueológicas, la cual únicamente condicionaba éstas a la autorización del Ejecutivo; un año más tarde se publicó la primera ley federal sobre la materia, la Ley sobre Monumentos Arqueológicos de 1897 (Sánchez, 2012).

Desde 1914 y hasta la fecha, en México se han promulgado cinco ordenamientos jurídicos cuyo objeto central ha sido la conservación de su patrimonio cultural:

1. "Ley sobre Conservación De Monumentos Históricos y Artísticos y Bellezas Naturales".

2. "Ley sobre Protección y Conservación de Monumentos y Bellezas Naturales".

3. "Ley sobre Protección y Conservación de Monumentos Arqueológicos e Históricos,

Poblaciones Típicas y Lugares de Belleza Natural".

4. "Ley Federal del Patrimonio Cultural de la Nación".

5. "Ley Federal Sobre Monumentos y Zonas

Arqueológicos, Artísticos e Históricos". (Ortiz, 2017)

Los ordenamientos jurídicos anteriormente citados nos dan cuenta de la relevancia que el patrimonio cultural ha cobrado en el interés nacional, como un elemento fundamental de la identidad de nuestra sociedad, de tal suerte esta cultura que ha sido forjada como parte de nuestra identidad, legalmente se ha consagrado a nivel constitucional al ser el acceso a la cultura un derecho de todos los mexicanos plasmado el artículo 4to de la Constitución Política de los Estados Unidos Mexicanos. Así mismo México es miembro de la Convención del Patrimonio Mundial de la UNESCO, lo que le confiere responsabilidades como nación para proteger el patrimonio cultural tanto material como inmaterial, para ser 
preservado no solo en beneficio de la nación si no de toda la humanidad.

El antecedente internacional inmediato de nuestra legislación vigente a nivel nacional en la materia, es el documento denominado como la Carta de Venecia suscrita en 1964 por los participantes en el del II Congreso Internacional de Arquitectos y Técnicos de Monumentos Históricos. En dicho documento se establecieron lineamientos del orden general de los cuales se fueron derivando los distintos ordenamientos jurídicos que norman la conservación y protección del patrimonio y que actualmente se encuentran vigentes, no solamente en nuestro país sino a lo largo del globo:

Las obras monumentales de los pueblos continúan siendo en la vida presente el testimonio vivo de sus tradiciones seculares. La humanidad, que cada día toma conciencia de la unidad de los valores humanos, los considera como un patrimonio común, y de cara a las generaciones futuras, se reconoce solidariamente responsable de su salvaguarda. Debe transmitirlos en toda la riqueza de su autenticidad. (1964)

Partiendo de los dispuesto en la Constitución mexicana, las intenciones manifestadas en la "Carta de Venecia" así como el consejo de distintos expertos en la materia, el poder legislativo federal discutió y aprobó la Ley Federal del Patrimonio Cultural de la Nación promulgada el 23 de diciembre de 1968, la cual fue sustituida en mayo de 1972 por la casi idéntica Ley Federal Sobre Monumentos y Zonas Arqueológicos, Artísticos e Históricos, la cual con sus adiciones y reformas actualmente continua en vigencia.

DE LOS CRITERIOS 
Analizando la Ley Federal Sobre Monumentos y Zonas Arqueológicos, Artísticos e Históricos, se aprecia que el patrimonio cultural tangible tanto los bienes inmuebles como muebles son definidos como "monumentos" a su vez dichos monumentos son divididos en tres categorías; arqueológicos, históricos y artísticos. Para dicha división, la legislación de 1972 utilizó los siguientes criterios:

\section{Monumentos arqueológicos}

Básicamente lo que la ley define como monumentos arqueológicos son aquellos bienes muebles o inmuebles producto de las civilizaciones prehispánicas, aunque dentro de la definición literal en la ley podemos encontrar una de las primeras ambigüedades, ya que solo se refiere textualmente a: "culturas anteriores al establecimiento de la hispánica" por lo cual la interpretación de dicho artículo puede ser subjetiva ya que no hace referencia sí se trata de un criterio de temporalidad o un criterio cualitativo. De ahí en más dentro de la misma categoría de monumento arqueológico se incluyen los restos de carácter paleontológico o antropológico, además de los bienes producidos por el ser humano que se encuentren sumergidos en el océano, es decir el patrimonio subacuático. (Ley Federal sobre Monumentos y Zonas Arqueológicas, Artísticos e Históricos, 1972, Art. 28)

\section{Monumentos históricos}

Para los monumentos históricos la legislación federal contempla dos esquemas, por declaratoria $\mathrm{O}$ por determinación de la ley; por la declaratoria correspondiente, podrán ser declarados monumentos históricos los que cumplan con las características de ser "bienes vincu- 
lados con la historia de la nación, a partir del establecimiento de la cultura hispánica en el país" (Ley Federal sobre Monumentos y Zonas Arqueológicas, Artísticos e Históricos,1972, Art. 35).

Por determinación de la Ley son monumentos históricos: "Los inmuebles construidos en los siglos XVI al XIX, destinados a templos y sus anexos; arzobispados, obispados y casas curales; seminarios, conventos etc., los inmuebles destinados al servicio y ornato públicos y al uso de las autoridades civiles y militares así como las obras civiles relevantes de carácter privado realizadas de los siglos XVI al XIX." De igual forma se incluyen dentro de la categoría los acervos documentales que cumplan con el criterio de temporalidad mencionado" (Ley Federal sobre Monumentos y Zonas Arqueológicas, Artísticos e Históricos, 1972, Art. 36).

\section{Monumentos artísticos}

En las definiciones de las categorías anteriores es observable la utilización de criterios cuantitativos, sin embargo en lo que a monumentos artísticos se refiere la ley hace uso únicamente de criterios cualitativos no exentos de ambigüedades; ya que la ley lo único que menciona como criterio que un bien debe cumplir para incluirse dentro de esta categoría es el "valor estético relevante" ante lo cual puede resultar en cientos de interpretaciones subjetivas sobre lo que represente o signifique ese valor estético. Citando textualmente la ley la definición sobre monumentos artísticos que arroja es la siguiente: los bienes muebles e inmuebles que revistan valor estético relevante. Y para considerarse con ese valor estético relevante es necesario que reúnan las características de: representatividad, inserción en determinada 
corriente estilística, grado de innovación, materiales y técnicas utilizados (SIC) y otras análogas (Ley Federal sobre Monumentos y Zonas Arqueológicas, Artísticos e Históricos, 1972, Art. 33).

\section{SituACIÓN DEL PATRIMONIO ARTístico}

Ahora bien, analizadas las categorías de monumentospatrimonio que define la legislación federal, el patrimonio artístico es el que a todas luces cuenta con la definición más ambigua dadas sus características, sería difícil definirlo por criterios meramente cuantitativos como la temporalidad, por lo cual solo es posible establecer un parámetro cualitativo, por lo tanto su estatus patrimonial solo es posible establecerlo a través de valoraciones realizadas por las autoridades competentes; lo que conduce a otra problemática los vacíos de autoridad a nivel federal derivados de lo establecido en la misma ley.

Para la valoración e inscripción de los monumentos pertenecientes al patrimonio arqueológico e histórico la ley faculta al Instituto Nacional de Antropología e Historia (INAH) conforme a los términos ya establecidos; en lo que al patrimonio artístico respecta, la competencia recae sobre el Instituto Nacional de Bellas Artes (INBAL) a través de la Comisión Nacional de Zonas y Monumentos Artísticos (Ley Federal sobre Monumentos y Zonas Arqueológicas, Artísticos e Históricos, 1972, Art. 34).

Aquí surgen los primeros detalles que favorecen un estado de precaria protección legal del patrimonio artístico, concretamente en las atribuciones de las instituciones competentes, mientras en el caso de los bienes de carácter arqueológico e histórico el Instituto Nacional de Antropología está debidamente facultado por ley para registrar, identificar, investigar, recuperar, rescatar, proteger, 
restaurar, rehabilitar, vigilar y custodiar dichos bienes. (Ley Orgánica del Instituto Nacional de Antropología e Historia, 1939, Art. $3^{\circ}$ ) Lo anterior en cuanto a la facultad de acción en cuanto a la capacidad de igual forma se le otorga al Instituto la facultad de crear delegaciones y centro regionales; ejemplo de esta situación es la presencia del Centro INAH en Aguascalientes ya con varias décadas de presencia en la entidad y contando con más de 1,400 monumentos históricos tan sólo en Aguascalientes.

Sin embargo las amplias facultades del INAH se ven limitadas por los criterios de competencia y definiciones de patrimonio, por lo cual en lo que respecta a las creaciones realizadas a partir del año 1900 son competencia del Instituto Nacional de Bellas Artes y Literatura (INBAL), el cual cuenta con atribuciones muy limitadas, en primer lugar debido a que la protección legal de los monumentos artísticos a diferencia de los históricos no surte efecto por determinación de la Ley si no por declaratoria previa ${ }^{1}$ y en segundo lugar debido a que la Ley Orgánica del INBAL no contempla la existencia de representaciones estatales o regionales; esto anterior sumado a las limitaciones técnicas y de personal han generado que una gran cantidad de bienes con valor cultural acorde a su contexto queden en un estado de desprotección absoluto, ya que ni siquiera se encuentran catalogados, dado que conforme a la ley para obtener la categoría de monumento artístico puede ser cualquier bien mueble o inmueble de valor estético relevante.

\footnotetext{
${ }^{1}$ Dice el Art. 34. de la Ley Federal sobre Monumentos y Zonas Arqueológicas, Artísticos e Históricos: "...la Comisión Nacional de Zonas y Monumentos Artísticos, la que tendrá por objeto dar su opinión a la autoridad competente sobre la expedición de declaratorias de monumentos artísticos y de zonas de monumentos artísticos. La opinión de la comisión será necesaria para la emisión de las declaratorias."
} 
Ante tal parámetro estético la cantidad de bienes con potencial de reunir tales características, resulta inconmensurable. Por otro lado, el proceso para su catalogación es a través del dictamen realizado por la Comisión Nacional de Zonas y Monumentos Artísticos, el cual no está exento de limitaciones; actualmente el Instituto en lo que a bienes muebles se refiere, únicamente tiene declarado como monumentos artísticos la obra de nueve artistas los cuales son José María Velasco, Diego Rivera, José Clemente Orozco, Gerardo Murillo "Dr. Atl", David Alfaro Siqueiros, Frida Kahlo, Saturnino Herrán, Remedios Varo y María Izquierdo (INBAL, 2018).

La situación de los inmuebles no es mejor, derivado de lo antes expuesto, como los criterios de definición legales ambiguos aunado a la centralización institucional y las mismas limitaciones orgánicas del INBAL; la inmensa mayoría de los inmuebles edificados a partir del siglo XX y con cierto valor estético y cultural, situados a lo largo y ancho del territorio nacional se encuentran en gran medida desprotegidos; en la mayoría de los casos ni siquiera catalogados ni registrados, carentes por completo de normativa que garantice su conservación. Los monumentos dependen únicamente de la protección que las autoridades locales y los mismos particulares les otorguen.

El problema que se suicita de competencias institucionales entre ambos institutos, sobre la responsabilidad sobre los monumentos arqueológicos e históricos por un lado y los artísticos por el otro, ha generado que los segundos se encuentren en un estado de protección precario (Proceso, 2003) esto solo en la Ciudad de México; pero en el interior del país considerando que el INBAL no cuenta con centros regionales, ni delegaciones estatales; la desprotección por parte de la autoridad federal se agudiza, por lo cual es necesario que las 
autoridades locales jueguen un papel importante para suplir las carencias operativas y legales del orden federal.

\section{EL MARCO LEGISLATIVO LOCAL EN EL ESTADO DE AGUASCALIENTES.}

A nivel local cada entidad enfrenta realidades muy distintas, ya sea por la difusión y naturaleza de sus bienes culturales con carácter patrimonial, las legislaciones estatales y reglamentos municipales, así como la presencia de las instituciones federales.

En Aguascalientes hay dos realidades bien diferenciadas la de los bienes considerados monumentos arqueológicos e históricos conforme a lo dispuesto en la ley federal, y la de los bienes creados a partir del año 1900 los cuales se clasificarían como monumentos artísticos, aunque solo unos pocos se encuentran con la declaratoria correspondiente.

En lo que refiere a los monumentos arqueológicos e históricos se encuentran bajo el amparo de la ley federal, además es de gran utilidad la presencia del Centro INAH Aguascalientes y el Museo Regional de Historia (dependiente del mismo instituto). De igual forma se han logrado avances considerables por parte del instituto al realizar colaboraciones con las instituciones locales del orden estatal y municipal; producto de ello tenemos proyectos como lo es el Taller de Restauración y Conservación del Instituto Cultural de Aguascalientes, estos proyectos han permitido generar un sistema cada vez más completo de protección al patrimonio anterior al siglo XX.

Sin embargo la situación de los bienes producidos a partir de 1900 es diametralmente distinta, salvo pocas excepciones no se cuenta con declaratorias de monumentos artísticos en el estado, por lo cual una gran 
cantidad de bienes de gran valor no cuentan con la protección de la ley federal; legalmente solo se cuenta a nivel local con la Ley de Protección y Fomento al Patrimonio Cultural publicada en el Periódico Oficial del Estado, el 2 de julio de 2001; si bien la ley tiene como objetivo general: la investigación, rescate, restauración, protección, conservación, registro, promoción y difusión del patrimonio cultural del Estado (Ley de Protección y Fomento del Patrimonio Cultural del Estado de Aguascalientes, 2001, Art. $3^{\circ}$ ) la promulgación de esta ley constituyó un paso importante para establecer mecanismos de protección al patrimonio cultural, contemplando un esquema de colaboración interinstitucional, competencias e incluso sanciones; en la práctica ha resultado un paso inconcluso derivado de varias deficiencias contenidas en la misma ley.

Las deficiencias son apreciables desde el comienzo del documento, en su artículo $4^{\circ}$ establece para que un bien legalmente cuente con el estatus de protección, deberá antes haberse realizado la declaratoria correspondiente, por lo cual sin la declaratoria no existe ningún esquema de protección que surta efecto por los términos de la ley. Otra deficiencia importante es la ambigua definición y categorización de los tipos de patrimonio cultural; aún más ambigua que la definición contenida en el marco legislativo federal. A nivel estatal se define dentro de la ley como bienes o monumentos artísticos sujetos a protección, a aquellos que posean valor estético (Ley de Protección y Fomento del Patrimonio Cultural del Estado de Aguascalientes, 2001, Art. 5). Continuando con las deficiencias de la ley estatal, en lo relativo a las competencias institucionales, no se establecen mecanismos claros para la aplicación de la misma; ya que atribuye competencias a distintas ramas de la administración 
pública, desde dependencias federales, los ayuntamientos y una variedad de dependencias del ejecutivo estatal como el Instituto Cultural de Aguascalientes, la Secretaría de Turismo, el Instituto de Educación e inclusive la desaparecida Secretaría de Planeación y Desarrollo Regional. Esta situación estipulada en la misma ley únicamente logra dificultar el deslinde de responsabilidades diluyendo las competencias por ende las distintas líneas de acción.

Los vacíos y deficiencias de la misma ley se agravan ante una falta de voluntad política que ha permeado en las sucesivas administraciones y legislaturas desde su promulgación; ya que desde hace más de 17 años que lleva la ley de haber sido promulgada aún no se ha publicado el reglamento respectivo para la aplicación de la norma. Dentro de la citada ley se contempla la creación de un Consejo Consultivo de Protección y Fomento del Patrimonio Cultural del Estado, el cual sería el encargado de delinear las políticas de acción en la materia y emitir declaratorias, A pesar de estar conformado y presidido por el Gobernador del Estado, por esa misma falta de voluntad política el consejo no ha sesionado en alguna ocasión hasta la fecha (Acero, 2014).

\section{CONCLUSIONES}

Aguascalientes se fundó en el ya lejano siglo XVI, sin embargo durante el periodo virreinal y gran parte del México independiente, fundada originalmente como la villa de las Aguas Calientes y posteriormente elevada a ciudad, la cual no experimentó un desarrollo tan vertiginoso como el que experimentó hasta finales del siglo XIX y todo el s. XX. El desarrollo experimentado a partir de la llegada del ferrocarril en el año de 1884 marca un 
antes y un después en la historia y cultura hidrocálida, junto con la primer locomotora que se asomó en el horizonte hidrocálido llegó la modernidad y la industrialización de la entidad (Terán, 2006).

La llegada de una nueva era en el porvenir de la ciudad generó a su vez la problemática de adaptar una población sustentada en las actividades agrícolas hacia una realidad urbana en continua expansión territorial, cultural y social. La ciudad inició un proceso de transformación que se vio reflejada en su traza urbana y arquitectura, con la llegada de nuevos materiales constructivos, técnicas y estilos se dio paso a la modernidad (López, 2005).

Desde el afrancesamiento y el eclecticismo porfiriano la fisionomía de la ciudad se fue transformando inicialmente de la mano del arquitecto empírico Refugio Reyes Rivas cuya gran parte de su obra data de los primeros años del siglo XX (Guajardo, 2013).

Con la apertura de la calle Francisco I. Madero en 1914 que conectaba el primer cuadro de la ciudad con la estación del ferrocarril, se abrió un espacio que fungió en las primeras décadas del siglo XX como un escaparate de la modernidad, por lo cual un gran número de edificaciones de estilos pertenecientes a la primer mitad del siglo, desde eclécticas, neocoloniales, art decó, funcionalistas etc. Fueron adornando la avenida y las calles aledañas como la calle González Saracho, desde viviendas, cines, departamentos y comercios estas edificaciones que fueron constituyendo un auténtico distrito moderno dentro de la ciudad de Aguascalientes. En las Figuras 1 y 2 se aprecian las distintas corrientes estilísticas de las edificaciones. 


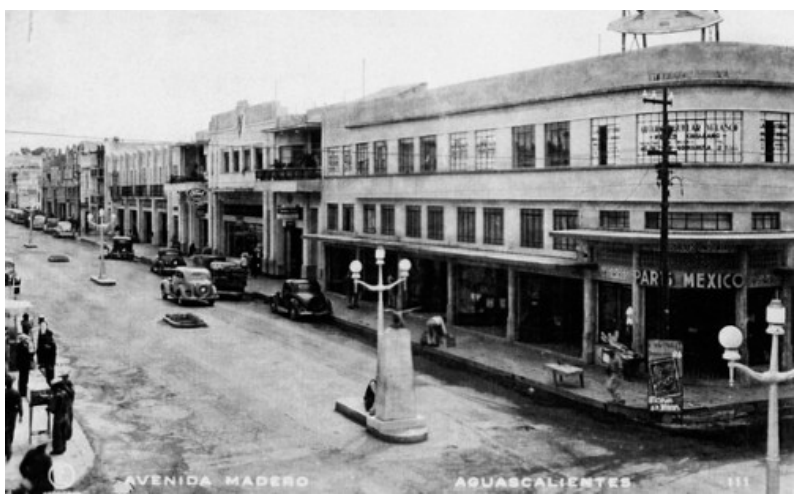

Figura 1. Av. Francisco I. Madero hacia el oriente durante la década de 1940. Fuente: Archivo Histórico del Estado.

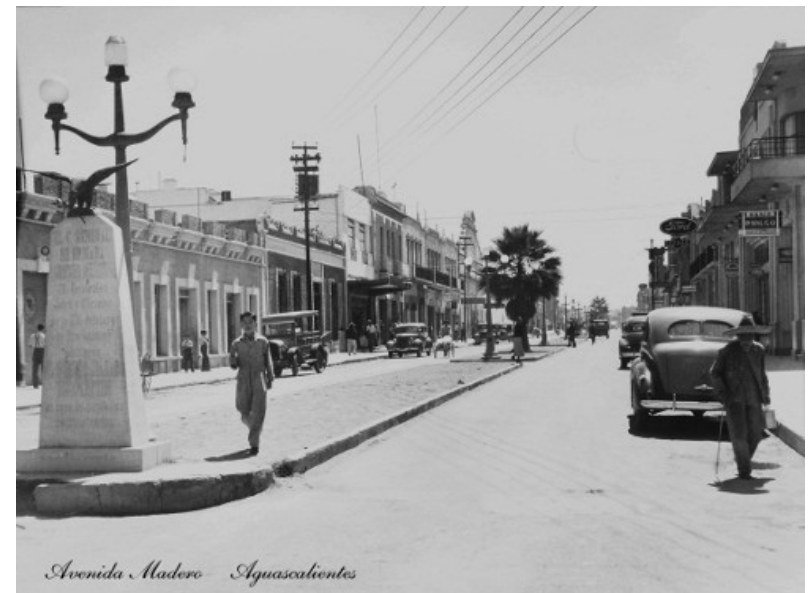

Figura 2. Av. Francisco I Madero hacia el poniente durante la década de 1940. Fuente: Archivo Histórico del Estado.

No solo la avenida Madero sino la ciudad entera sufrió la transformación más grande en su haber, no solamente en su fisionomía: los cambios tecnológicos, sociales y 
culturales fueron muy acelerados en todo el siglo XX, lo que forjó la identidad cultural de la capital hidrocálida. Testimonio de la construcción del Aguascalientes moderno, actualmente sobreviven una gran cantidad de edificaciones, cuyo valor estético, histórico y cultural es incalculable, sin embargo al paso de los años cada vez son menos las edificaciones que se mantienen en pie debido a que no cuentan con la protección de las leyes ni de las instituciones. Por citar un caso, es sabido que más de la mitad de la obra del arquitecto práctico Refugio Reyes Rivas data de 1900 en adelante, por lo cual se encuentra sin protección legal ni institucional (Guajardo, 2013), como se ilustra en la Figura 3.

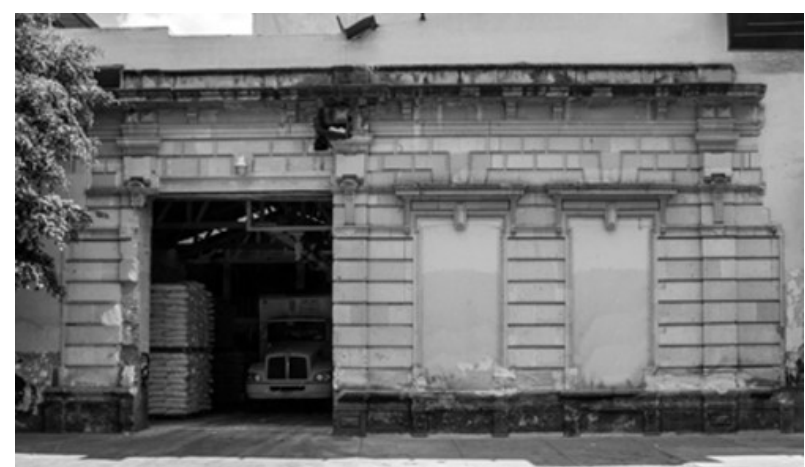

Figura 3. Molino San Marcos sobre los restos de la Casa de Carlos Doerr, obra de Refugio Reyes del año de 1909.

Es una realidad complicada de cambiar desde el plano local; las deficiencias legales e incapacidad de las autoridades federales para proteger el patrimonio cultural y artístico del siglo XX, la clasificación de los monumentos en la ley federal las cuales fueron calificadas como absurdas por el experto en la materia Bolfy Cottom 
(Proceso, 2003) y la misma obsolescencia de la ley federal la cual esta próxima a cumplir 50 años de ser promulgada sin recibir actualizaciones relevantes. Teniendo en cuenta esta situación es posible adecuar el marco legal a nivel estatal, con la finalidad de llenar los vacíos, corregir las situaciones y supuestos que se han mencionado.

Las instituciones estatales y municipales en Aguascalientes, han demostrado tener la capacidad de hacerse cargo del problema, prueba de ello son acciones y proyectos que se han implementado con gran éxito en los distintos niveles de gobierno, desde la Dirección de Restauración de Monumentos que estuvo adscrita a la Secretaría de Obras Públicas, dirección hoy desaparecida, programas municipales como el Restaura y Revalora (SOPMA, 2018) en cuanto a bienes inmuebles, y en cuanto a bienes muebles el reciente proyecto exitoso y ambicioso del Taller de Conservación y Restauración del Instituto Cultural de Aguascalientes, por citar algunos ejemplos que han sido de gran ayuda para conocer y conservar el patrimonio. Sin embargo ante la carencia de un marco legal que respalde dichos proyectos exitosos suelen carecer de continuidad ante los cambios de administración y otros factores de índole política, sin una ley que norme las acciones en la materia se cae en el riesgo de realizar proyectos controversiales y desafortunados como lo fueron la construcción del mausoleo de Jesús F. Contreras en el Museo Aguascalientes, o las demoliciones que recientemente se hicieron en las inmediaciones del Teatro Morelos (ver Figura 4) solo por citar algunos ejemplos.

Gran parte del patrimonio cultural y artístico del estado se encuentra bajo esta situación de absoluta desprotección. La misma legislación estatal contempla un registro público del patrimonio estatal que a la fecha sigue sin ser implementado, por lo cual los objetivos para los 
que fue creada la Ley de Protección y Fomento al Patrimonio Cultural del Estado de Aguascalientes no han sido cumplidos.

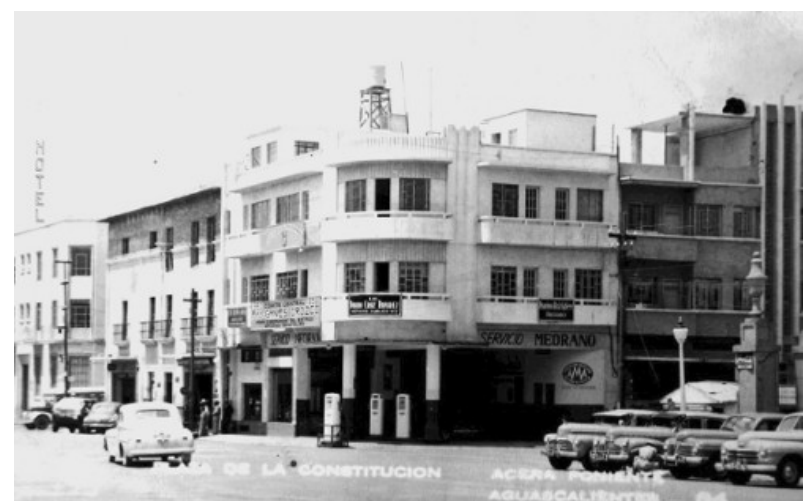

Figura 4. Edificio de usos mixtos estilo Art Déco ubicado en la actual plaza de Jacarandas en Aguascalientes

Fuente: Archivo Histórico del Estado.

Se requiere de una revisión de las leyes, reglamentos y estatutos, de manera profunda basada en un estudio sustentado y a conciencia, libre de motivaciones políticas que permitan realizar los cambios necesarios para revertir la situación actual. Legislar con base a la realidad local y no simplemente copiar legislaciones sin analizar; por citar un ejemplo desafortunado, el estado de Tabasco cuenta con una la Ley de Protección y Fomento al Patrimonio Cultural, la cual es prácticamente idéntica a la ley de Aguascalientes, dos estados con realidades tan distintas no pueden tener la misma normativa.

Sin embargo también hay ejemplos exitosos de entidades que se encuentran bastante avanzadas en la materia de legislación cultural uno de ellos es el vecino 
estado de Zacatecas, cuya Ley de Protección y Conservación del Patrimonio Cultural del Estado, promulgada en 2014, contiene una definición mucho más compleja y adecuada del patrimonio. Asimismo, dicha ley contempla competencias bien definidas y la creación de una dependencia especializada en el ramo como lo es la Junta de Protección y Conservación de Monumentos y Zonas Típicas del Estado de Zacatecas. Estos ejemplos pueden ser utilizados para ser replicados de manera exitosa en Aguascalientes.

La importancia de la necesidad de un nuevo marco jurídico en la materia ha quedado más que clara, mientras la situación actual perviva se seguirán perdiendo bienes de gran valor artístico y cultural, y las medidas de conservación a merced de la discrecionalidad de políticos y particulares.

Se requiere de una legislación que identifique y registre adecuadamente los bienes con valor artístico y cultural en el estado, que no se encuentren bajo la protección de la ley federal; una ley que faculte y descargue adecuadamente las competencias en las instituciones para su vigilancia, conservación y restauración. Además la ley debe atender la significación del patrimonio cultural para con la sociedad, que involucre a las comunidades, instituciones educativas, colegios de profesionistas y ayuntamientos; que genere esquemas adecuados de gestión; que incentive a los propietarios para su conservación. Estos objetivos son posibles de alcanzar, objetivos que además se traducen en grandes beneficios para la sociedad y el turismo en la entidad. 
Acero, I. (31 de agosto de 2014). Dictaminan sin leer cambios a la ley de protección y fomento al patrimonio cultural. La Jornada Aguascalientes. Recuperado de https://www.lja.mx/2014/08/dictaminan-sin-leercambios-a-la-ley-de-proteccion-y-fomento-alpatrimonio-cultural/

Cottom, B. (2008). Nación, Patrimonio Cultural y Legislación: los debates parlamentarios y la construcción del marco jurídico federal sobre monumentos en México, siglo XX. México, D.F: Porrua.

Guajardo, P. (2013). Refugio Reyes Rivas, Arquitecto empírico. Aguascalientes: Instituto Cultural de Aguascalientes.

Carta internacional sobre la conservación y la restauración de monumentos y sitios. (1964). Recuperado de https://www.icomos.org/charters/venice sp.pdf

INBAL. (2018). Obras con declaratoria de monumento artístico. Instituto Nacional de Bellas Artes y Literatura. Recuperado de https://www.inba.gob.mx/transparencia/obras

Llull, J. (2005). Evolución del concepto y de la significación social del patrimonio cultural. Arte, Individuo y Sociedad, 17. 175-204. Recuperado de https://revistas.ucm.es/index.php/ARIS/article/view/66 $\underline{56}$

López, J. J. (2005). Arquitectura de Aguascalientes. La primera mitad del siglo XX [Tesis doctorall. Colima: Universidad de Colima.

Ley de Protección y Fomento del Patrimonio Cultural del Estado de Aguascalientes. (2 de julio del 2001). Recuperado de 
http://eservicios2.aguascalientes.gob.mx/NormatecaAd ministrador/archivos/EDO-18-52.pdf

Ley Federal sobre Monumentos y Zonas Arqueológicas,

Artísticos e Históricos. (6 de mayo de 1972).

Recuperado de

http://www.diputados.gob.mx/LeyesBiblio/pdf/131 16

0218.pdf

Ley Orgánica del Instituto Nacional de Antropología e

Historia. (3 de febrero de 1939). Recuperado de https://

mexico.justia.com/federales/leyes/ley-organica-del-

instituto-nacional-de-antropologia-e-historia/gdoc/

Moreno, C. (2019). Reflexiones sobre memoria y patrimonio de

nuestra tierra, nuestra gente y su cultura. Buenos Aires:

ICOMOS ARGENTINA. Recuperado de

http://todopatrimonio.com/2019/06/08/reflexiones-

memoria-patrimonio-nuestra-tierra-nuestra-gente-

cultura/

Ortiz, A. D. (24 de octubre de 2017). Iniciativa con proyecto de decreto por el que expide la ley federal del patrimonio cultural de la nación. Gaceta Parlamentaria.

México. Recuperado de http://sil.gobernacion.gob.mx/

Archivos/Documentos/2017/11/asun 3636451201711

30 1512409776.pdf

Proceso. (2003). El patrimonio del siglo XX, tierra de nadie.

Proceso. Recuperado de

https://www.proceso.com.mx/189115/el-patrimonio-

del-siglo-xx-tierra-de-nadie

Sánchez, L. (2012). Legislación mexicana del patrimonio

cultural. Derechos culturales, Cuadernos Electrónicos, 8, 57-74.

Recuperado de

https://pradpi.es/es/publicaciones/cuadernos-

electronicos/cuaderno-electronico-8-2012 
SOPMA. (2018). Programa Revalora. Aguascalientes:

Ayuntamiento de Aguascalientes.

Terán, E. (2006). Aguascalientes. Sus barrios desde la tradición oral. Aguascalientes: Ayuntamiento de Aguascalientes.

\section{ACERCA DEL AUTOR}

José Juan Hernández Moncada es docente de Museografía y Museología e Historia del Arte en la Universidad de las Artes. Historiador por la Universidad Autónoma de Aguascalientes, actualmente desempeñando como docente en la Universidad de las Artes y la Universidad Autónoma de Durango, colaborador en la investigación para el libro Refugio Reyes Arquitecto Empírico. Alumno inscrito en la maestría de Conservación y Restauración del Patrimonio Edificado del ICA (ORCID 0000-0001-62680708).

Recibido: 08/11/2018 Aceptado: 22/03/2019

CÓMO CITAR ESTE ARTÍCULO

Hernández Moncada, J. J. (2020). La precaria protección del patrimonio cultural del siglo XX en Aguascalientes.

Caleidoscopio - Revista Semestral de Ciencias Sociales y Humanidades, 23(42), 25-49. doi:10.33064/42crscsh2150 